Trauma Berufskrankh 2006 · 8 [Suppl 1]: S14-S19

DOI 10.1007/s10039-006-1110-6

Online publiziert: 23. Februar 2006

(c) Springer Medizin Verlag 2006

J. von Recum · H. Mayer · K. Wendl · P. A. Grützner · A. Wentzensen

BG-Unfallklinik, Unfallchirurgische Klinik, Universität Heidelberg, Ludwigshafen

\title{
Frische Verletzung des OSG
}

bandapparat dreiteilig aufgebaut und unterteilt in

- einen vorderen Zügel (Lig. fibulota-

lare anterius),

- einen mittleren Zügel (Lig. fibulocalcaneare) und

- einen hinteren Zügel (Lig. fibulotalare posterius).

Das Lig. deltoideum als mediales Seitenband besteht aus einer oberflächlichen und einer wesentlich kräftigeren tiefen Schicht.

\section{Verletzungsanalyse}

Verletzungen des Fußes und oberen Sprunggelenks stellen mit $10-15 \%$ einen erheblichen Anteil des Patientenguts unfallchirurgischer Kliniken dar. Nach Ochs et al. [10] beträgt die Inzidenz für Sprunggelenkfrakturen im europäischen Raum 1:100o Einwohner.

Die typischen Mechanismen entsprechen indirekten Gewalteinwirkungen: Über 80\% der Verletzungen entstehen infolge von Verrenkungsmechanismen, bei etwa $10 \%$ sind Hochrasanztraumen ursächlich. Direkte Gewalteinwirkungen, z. B. in Form axialer Krafteinleitung, spielen bei der Entstehung von oberen Sprunggelenkverletzungen eine untergeordnete Rolle [20].

Die Verletzungsmorphologie ist abhängig von der Stellung des Fußes im Moment der Krafteinleitung sowie der Richtung des Kraftvektors. Aufgrund der hohen Formschlüssigkeit des Gelenkkomplexes und der straffen ligamentären Führung entstehen in vielen Fällen Kombinationsverletzungen der knöchernen und ligamentären Strukturen sowie des Gelenkknorpels.

Dem Unfallmechanismus sollte im Rahmen der Anamneseerhebung aufgrund obiger Ausführungen besondere Bedeutung beigemessen werden. Die klinische Untersuchung sollte neben den klassischen Untersuchungsmethoden die Überprüfung der ligamentären Stabilität beinhalten.

\section{Bildgebung}

\section{Röntgen}

Als Standard ist die Röntgenuntersuchung des oberen Sprunggelenks (OSG) in 2 Ebenen anzusehen. Zur Beurteilung der Stellung der Malleolengabel und der Torsion des Außenknöchels ist die um $20^{\circ}$ nach innen gedrehte a.-p. Aufnahme des OSG (mortise view) besser geeignet als die streng a.-p. ausgerichtete Aufnahme.

Je nach Fragestellung sollten diese Befunde durch weitere Projektionen und Belastungsaufnahmen erweitert werden: „Isolierte“ Frakturen des Innenknöchels in Folge eines Distorsionsereignisses lassen einen Abduktionsmechanismus vermuten. Hier muss die Röntgenuntersuchung um eine Projektion des Unterschenkels in 2 Ebenen zum Ausschluss

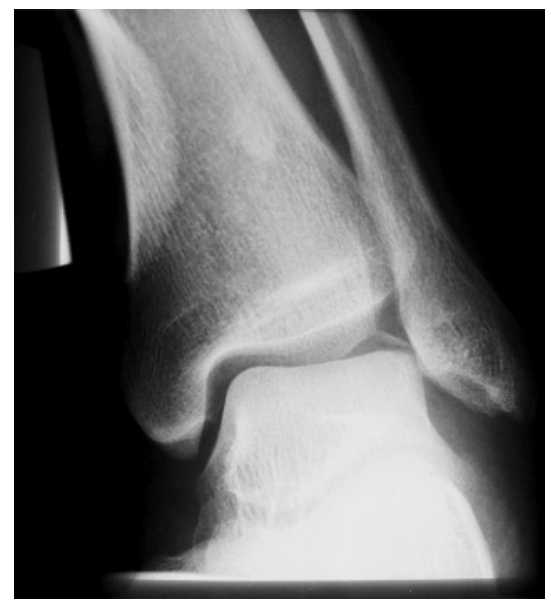

Abb. $1 \Delta$ Osteochondrale Fraktur laterale Talusschulter bei Außenbandruptur 


\section{Zusammenfassung $\cdot$ Abstract}

eines hohen Wadenbeinbruchs als Folge einer Pronations-Eversions-Fraktur ergänzt werden.

\section{Computertomographie}

Sie kommt nur bei bestimmten Fragestellungen zum Einsatz. Bei klinisch nicht sicher beurteilbaren Fällen führen wir in unserer Klinik computertomographische Messungen der Malleolengabel im Seitenvergleich zur Beurteilung der Integrität der distalen tibiofibularen Syndesmose durch.

\section{Magnetresonanztomographie}

Sie kann in unklaren Fällen fortdauernder Beschwerdesymptomatik ohne radiologischen Verletzungsnachweis Klärung bei okkulten Frakturen liefern. Darüber hinaus ist mit ihr eine gute Beurteilung der ligamentären Strukturen möglich.

\section{Verletzungen}

\section{Knorpel}

Verletzungen des Gelenkknorpels des OSG entstehen infolge von Verrenkungsmechanismen durch scherende Krafteinwirkung. Meist handelt es sich um Begleitschäden im Rahmen von Sprunggelenkfrakturen und Supinationsluxationen. Die Mehrzahl der Veränderungen finden sich an der lateralen Talusschulter ( $\bullet$ Abb. 1). Knorpelläsionen bedeuten in der Regel eine Prognoseverschlechterung.

Isolierte Knorpelverletzungen entgehen meist der primären Diagnostik und entziehen sich somit einer kausalen Therapie. Lang anhaltende posttraumatische Beschwerden und rezidivierende Blockierungen können auf freie Gelenkkörper infolge kartilaginärer Abschlagfragmente hinweisen. Demgegenüber lassen sich Flake-Frakturen (osteochondrale Abschlagfragmente) in den standardisierten Röntgenprojektionen darstellen. Hier besteht die kausale Therapie in der Gelenkrevision mit Refixation des Fragments.

\section{Bandstrukturen}

Verletzungen des Außenbandapparats des oberen Sprunggelenks gehören zu den häufigsten Verletzungen in Mitteleuropa.

Trauma Berufskrankh 2006 · 8 [Suppl 1]: S14-S19

DOI 10.1007/s10039-006-1110-6

(C) Springer Medizin Verlag 2006

J. von Recum · H. Mayer · K. Wendl · P. A. Grützner · A. Wentzensen

\section{Frische Verletzung des OSG}

\section{Zusammenfassung}

Vor Behandlung einer Sprunggelenkverletzung muss das gesamte Verletzungsausmaß erfasst werden. Dies ist nur bei entsprechenden Kenntnissen über die biomechanischen Grundlagen dieses komplexen Gelenks möglich. Bei der Diagnose kommt neben Anamnese und klinischer Untersuchung - dem konventionellen Röntgen eine wesentliche Bedeutung zu. In besonderen Fragestellungen haben Funktionsaufnahmen und Schnittbilddiagnostik ihren Stellenwert. Die Einteilung der Sprunggelenkfrakturen nach Lauge-Hansen verbessert das Verständnis der Verletzungsabfolge und gibt entscheidende Hilfestellungen bei der Erfassung der ligamentären Begleitverletzungen. Besonderer Beachtung bedürfen die hochgradig instabilen Verletzungen vom Pronations-Eversions-Typ. Hier ist die Wiederherstellung der Syndesmose und damit die Stabilität der

\section{Fresh ankle injury}

\section{Abstract}

The treatment of ankle injuries requires ascertainment of the total extent of the injury in each case. Knowledge of the basic biomechanical principles at play in this complex joint is essential. For the diagnosis, in addition to the history and the clinical examination, great importance also attaches to conventional X-ray examination. When there are particular problems with the diagnosis, functional $\mathrm{X}$-rays and $\mathrm{CT}$ are valuable adjuncts. Classification of ankle fractures according to the Lauge-Hansen system enhances understanding of the course of the injury and is definitely helpful in the ascertainment of associated ligamentous injuries. Highly unstable injuries of the pronation-eversion type need particular attention. In these cases restoration of the syndemosis, and thus of the stability of the malleolar fork, is essential if a functionally satisfactory result is to be at-
Malleolengabel Grundvoraussetzung für ein funktionell zufrieden stellendes Ergebnis. Die Einstellung der Fibula in die Inzisur ist technisch anspruchvoll, die Platzierung der Stellschraube beinhaltet Komplikationsmöglichkeiten. Die Überprüfung der exakten Einstellung ist mit dem konventionellen 2D-Bildverstärker nicht ausreichend sicher möglich. Hier ist ein Einsatz der Schnittbilddiagnostik zu fordern. Mit den 3D-Bildwandlern kann diese Überprüfung intraoperativ in ausreichend guter Qualität erfolgen. Damit wird der logistische Aufwand einer postoperativen CTDiagnostik reduziert, und Revisionseingriffe werden vermieden.

\section{Schlüsselwörter}

Sprunggelenkverletzung - Biomechanische Grundlagen · Einteilung nach Lauge-Hansen . 3D-Bildwandler · Syndesmoseverletzung

tained. It is technically difficult to insert the fibula in the incision, and placement of the positioning screw is also not without its pitfalls. It is not possible to check the precise alignment with an adequate level of certainty when a conventional 2D image intensifier is used. Diagnostic CT imaging should be prescribed for this purpose. Adequate quality can be achieved when this test is performed intraoperatively with a 3D image transformer. This makes it possible to avoid the logistical complications of postoperative diagnostic CT and also revision operations.

\section{Keywords}

Ankle injury - Biomechanical principles . Classification according to Lauge-Hansen system · 3D Image transformer - Tibio-fibular syndesmoses 


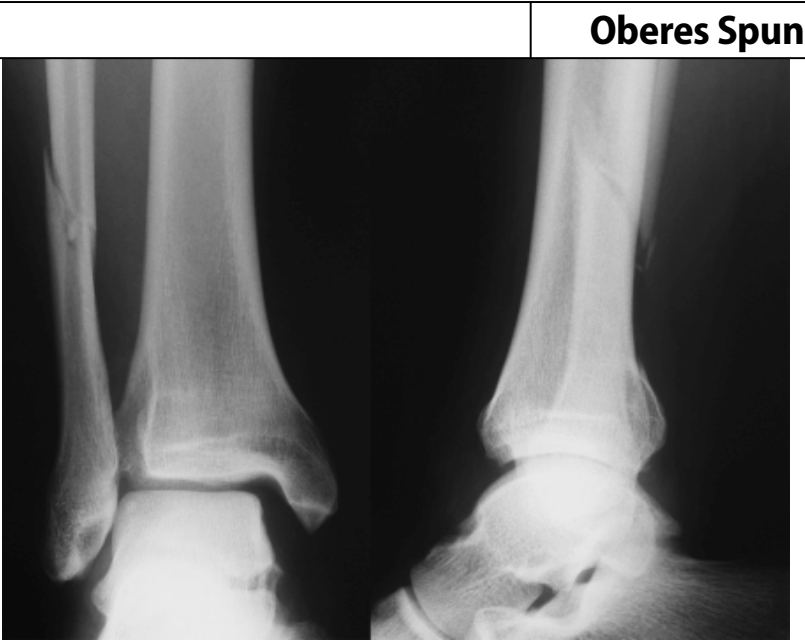

Abb. $2 \triangleleft$ Pronations-Eversions-Fraktur

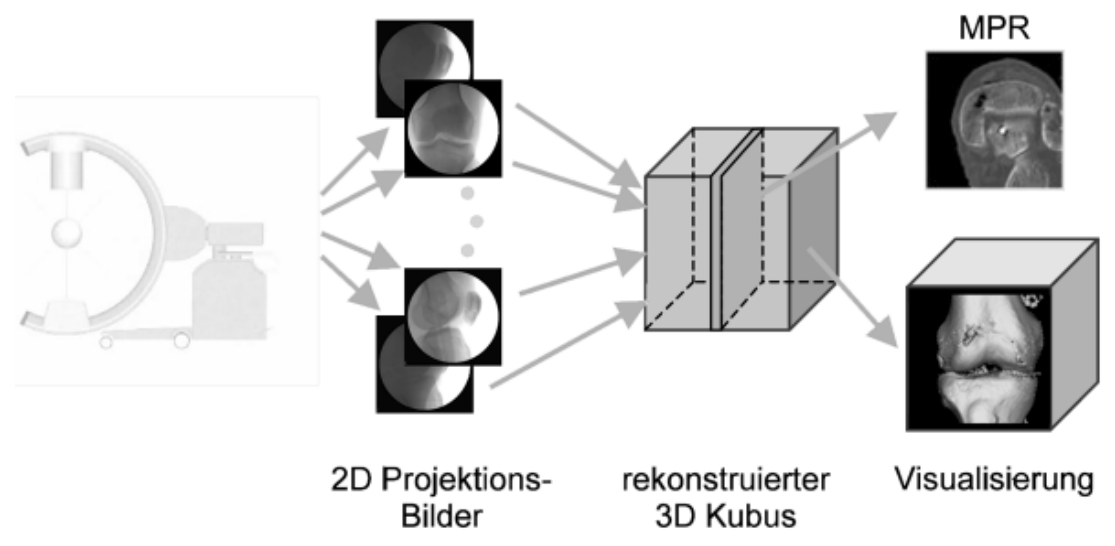

Abb. 3 \ Prinzip der intraoperativen 3D-Bildgeneration

Die vermehrte laterale Instabilität führt zu einer Verkippung des Talus um die Längsachse in varischer Richtung sowie einer Translation in a.-p. Richtung. Die fehlende Zügelung des Talus an die Außenknöchelspitze bewirkt darüber hinaus eine nach innen gerichtete Rotationskomponente, weswegen in letzter Zeit auch von einer „antero-lateralen Rotationsinstabilität“ (ALRI) gesprochen wird.

Aufgrund mehrerer prospektiv randomisierter Studien zur Behandlung der frischen Verletzung des Außenbandapparats des oberen Sprunggelenks [21] gilt die funktionelle Therapie der operativen Behandlung als ebenbürtig, unter Vermeidung der operationsbedingten Komplikationen.

Indikationen zur operativen Therapie bestehen noch bei extremen Formen der Instabilität im Sinne einer „Luxatio pedis cum talo“, osteochondralen Begleitläsionen, offenen Verletzungen oder Verletzungen mit drohender Weichteilschä- digung und irreponierbaren knöchernen Ausrissen [20].

Die „AG Fuß“ der Deutschen Gesellschaft für Unfallchirurgie plant derzeit die Auflage einer multizentrischen Studie zur Behandlung der 3-Band-Verletzungen des oberen Sprunggelenks.

Isolierte Verletzungen des medialen Kollateralbandes (Lig. deltoideum) stellen Raritäten dar. Grundsätzlich muss in diesen Fällen gezielt nach Verletzungen der tibiofibularen Syndesmose, des Außenknöchels und des lateralen Bandkomplexes gesucht werden. Isolierte Verletzungen werden in der Regel konservativ behandelt $[20,22]$, die Darstellung des Innenbandes und die Naht im Rahmen einer Sprunggelenkfraktur konnten im Rahmen einer prospektiv randomisierten Studie [15] keinen Vorteil im funktionellen Ergebnis zeigen.

\section{Frakturen}

\section{Klassifikation}

Einteilung nach Danis u. Weber [18]. Sie ist bei Sprunggelenkfrakturen die gebräuchlichste. Unter Orientierung der Höhe der Außenknöchelfraktur zur tibiofibularen Syndesmose werden die Typen A-C unterschieden.

- Typ Weber A: infrasyndesmale Verletzung, Syndesmosenkomplex intakt

- Typ Weber B: transsyndesmale Fraktur, Teilverletzung des Syndesmosenkomplexes, Malleolengabel nicht zwingend instabil

- Typ Weber C: suprasyndesmale Fraktur, vollständige Zerreißung aller Anteile der Syndesmose, generell instabil

Verletzungen des Innenknöchels, des Innenbandes und knöcherne Ausrisse der hinteren Syndesmosenanteile (hinteres Volkmann-Dreieck) finden in dieser Klassifikation keine Berücksichtigung.

AO-Klassifikation. Sie führt die Sprunggelenkverletzungen unter der Ziffer 44. Sie ergänzt die Einteilung nach Weber um die prognostisch und indikatorisch relevanten medialen Begleitverletzungen in den Untergruppen und hilft bei der Unterscheidung stabiler und instabiler Verletzungen [9].

Einteilung nach Lauge-Hansen. Ihr kommt besondere Bedeutung für das Verständnis der Frakturentstehung, die Beurteilung der Stabilität und die Wahl des Therapieverfahrens zu [7]. Sie beruht auf der Entstehung sich wiederholender spezifischer Verletzungsmuster als Folge der Richtung der eingeleiteten Kraft. Die Bezeichnung des Frakturtyps setzt sich aus

- der Stellung des Fußes (Supination/ Pronation) und

- der Richtung der eingeleiteten Kraft (Adduktion/Abduktion/Eversion)

zusammen. Lauge-Hansen [7] unterschied 4 Grundtypen:

- Supinations-Adduktions-Fraktur

- Supinations-Eversions-Fraktur

- Pronations-Abduktions-Fraktur

- Pronations-Eversions-Fraktur 


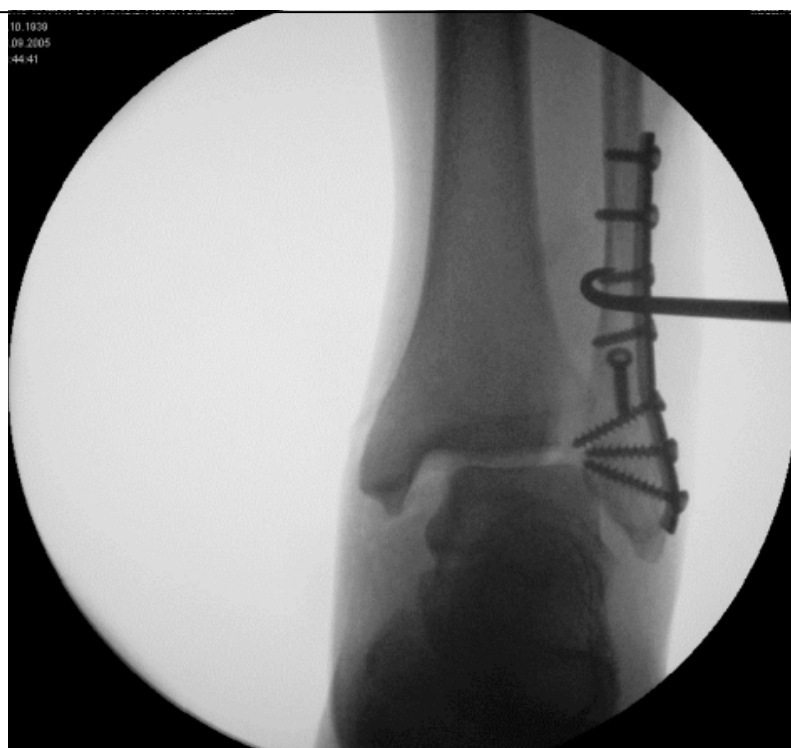

Abb. $4<$ Instabile Malleolengabel mit Subluxation des Talus nach lateral, erweitertem Espace claire nach Chaput und weitem „medial clear space”

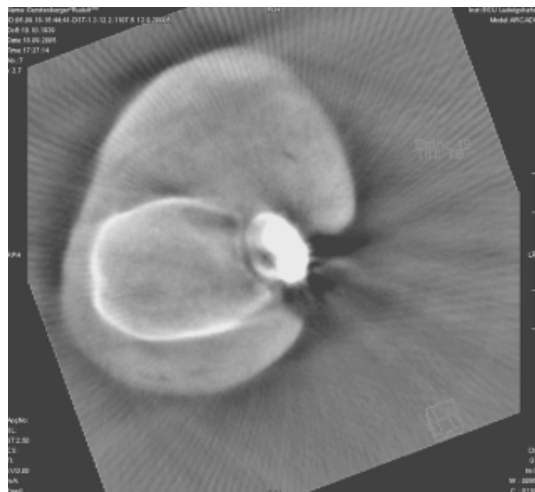

Abb. $6 \Delta$ Stellung der Fibula in der Inzisur in axialen Rekonstruktionen

Diese sind in weitere Subtypen unterteilt, die Stadien in der Verletzungsentstehung darstellen.

Während Supinations-AdduktionsFrakturen ohne Verletzung des Syndesmosenkomplexes einhergehen, entstehen im Rahmen des Pronations-Abduktionssowie des Supinations-Eversions-Mechanismus partielle Verletzungen der Syndesmose. In der Regel bleiben hierbei die interossären Anteile sowie die Membrana interossea erhalten, weswegen nach Reposition der Außen- und Innenknöchelfraktur keine relevanten Instabilitäten der Malleolengabel verbleiben.

Besonderer Beachtung bedürfen die Pronations-Eversions-Frakturen (• Abb.2). Durch die Außenrotation des Talus in Pronationsstellung des Fußes entsteht zunächst eine horizontale Innenknöchelfraktur im Sinne einer Abschlagverletzung. Die fortgeleitete Rotationskomponente führt im Anschluss zur

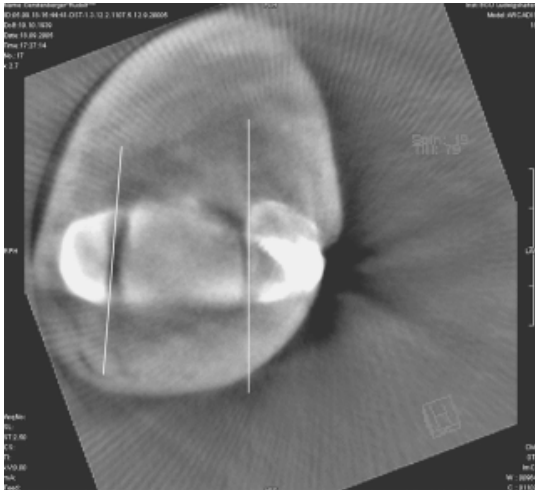

Abb. $7 \Delta$ Torsionsbestimmung in axialen Rekonstruktionen

Ruptur aller Anteile der Syndesmose und bewirkt damit eine vollständige Instabilität der Malleolengabel. Bei fortdauernder Krafteinleitung folgt die suprasyndesmale Fibulafraktur.

\section{Therapie}

Biomechanische Untersuchungen zeigen die besondere Bedeutung der Anatomie der Malleolengabel auf die Belastung des Sprunggelenks. Nach Thordarson [16] führt die Verkürzung der Fibula um $\geq 2 \mathrm{~mm}$ oder eine Außenrotation der Fibula $\geq 5^{\circ}$ zu einem signifikanten Druckanstieg. Die Lateralverschiebung des Talus um $1 \mathrm{~mm}$ als Folge eine Instabilität der Malleolengabel oder Fehlstellung des Außenknöchels führt zu einer Reduktion der tibiotalaren Kontaktfläche um $40 \%[13,17]$. Die Behandlung der Sprunggelenkfrakturen muss demnach die knöcherne Heilung in anatomisch korrekter

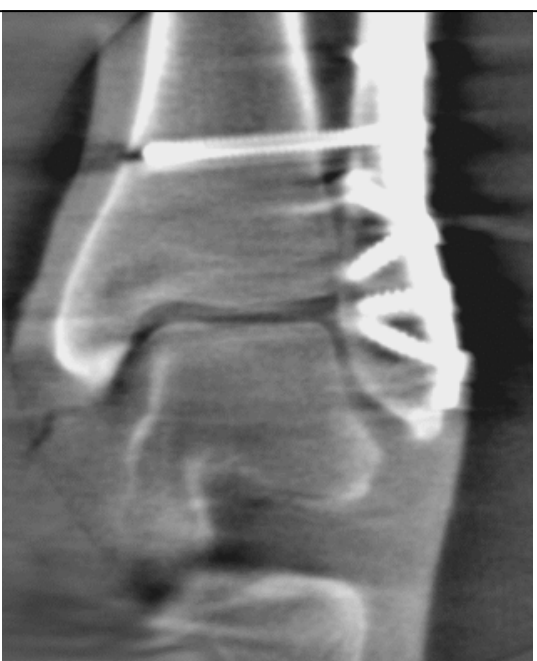

Abb. $5 \Delta$ Koronare Rekonstruktion zur Beurteilung der Länge der Fibula

Stellung bei stabilem ligamentärem Komplex gewährleisten.

Konservativ. Undislozierte Frakturen ohne begleitende ligamentäre Verletzungen und mit stabiler Malleolengabel können konservativ funktionell therapiert werden. In einer vergleichenden Untersuchung konnten Özokyay et al. [11] gute funktionelle Ergebnisse nach Behandlung undislozierter Weber-B-Frakturen mittels Aircast-Schiene nachweisen. Vorraussetzung für eine konservative Therapie ist der Ausschluss einer begleitenden Instabilität des Syndesmosenkomplexes.

Operativ. Die oben erwähnten biomechanischen Untersuchungen stellen die besondere Bedeutung der anatomischen Reposition und Wiederherstellung der funktionellen Einheit der Malleolengabel heraus. Dislozierte Frakturen bedürfen einer anatomischen Reposition. In der Regel ist dazu ein operativer Eingriff erforderlich. Ziel sollte die übungsstabile Retention sein, andernfalls muss das Operationsergebnis durch Immobilisation mittels Unterschenkelgips oder Gehorthese gesichert werden. Intraoperativ schließt sich die Überprüfung der Stabilität der Syndesmosenkomplexes an. Bewährt haben sich der Druck von ventral auf den Außenknöchel zur Überprüfung der vorderen Anteile und der lateral gerichtete Zug mit dem Einzinkerhaken zur Überprüfung der interossären Anteile. Persistiert nach Reposition einer SupinationsEversions- oder Pronations-Abduktions- 

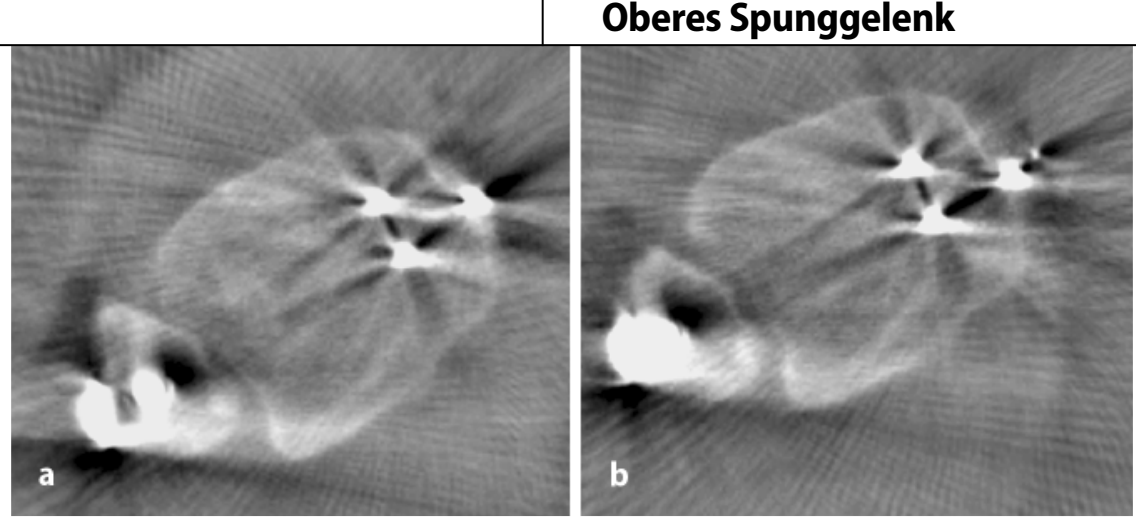

Abb. $8 \Delta$ Zu weit ventral in der Inzisur eingestellte Fibula (a) nach Korrektur (b)

Fraktur eine Instabilität der Syndesmose, sollte zunächst die Reposition des Außenknöchels auf Länge und Rotation erfolgen, da bei diesen Formen die wichtigen interossären Anteile in der Regel erhalten bleiben. Ist die Reposition regelrecht, muss die Syndesmose revidiert und bis zur Ausheilung retiniert werden.

Biomechanische Untersuchungen haben eine Ausreißfestigkeit für die isolierte Naht der vorderen Syndesmose von $2 \mathrm{Nm}$ gemessen. Eine trikortikale Stellschraube erhöht die Stabilität auf 6,2 Nm [19]. Sie gilt im Rahmen der Versorgung frischer Sprunggelenkverletzungen mit Syndesmoseninstabilität als "Goldstandard“ [2, $6,8,12]$.

In der nach Henkemeyer et al. [4] beschriebenen Technik transfixiert eine Kortikalisschraube mit durchgehendem Gewinde Fibula und Tibia etwa $2 \mathrm{~cm}$ oberhalb des Sprunggelenks. Dabei muss Wert darauf gelegt werden, die Schraube parallel zur Rotationsebene des oberen Sprunggelenks, also um $30^{\circ}$ aus der frontalen Ebene von dorsolateral nach anteromedial ansteigen zu lassen. Hilfreich ist in vielen Fällen die Verwendung einer ausladenden Repositionszange, die "tip to tip“ von der Außen- auf die Innenknöchelspitze gesetzt wird. Bei dieser Positionierung können im Gegensatz zu der häufig auf Höhe der Inzisur angelegten Repositionszange größere Torsionsabweichungen vermieden werden.

Die intraoperative Überprüfung der Frakturreposition gelingt gut in der Durchleuchtung in 3 Ebenen. Die sichere Beurteilung der Torsion und der Stellung der Fibula in der Inzisur ist nur im Rahmen der Schnittbilddiagnostik möglich. Dies macht bei Sprunggelenkverletzungen mit Beteiligung des Syndesmo- senkomplexes eine postoperative CT-Diagnostik erforderlich. Wird ein revisionsbedürftiger Stellungsbefund diagnostiziert, muss zum frühestmöglichen Zeitpunkt revidiert werden. Nach erfolgter Reosteosynthese wird eine erneute computertomographische Kontrolle erforderlich. Erst diese Vorgehensweise sichert korrekte Repositionsergebnisse, bedeutet aber einen enormen logistischen Aufwand und eine nicht zu unterschätzende Zahl an Revisionseingriffen.

Mit dem 3 D-Bildwandler steht seit einigen Jahren eine Technik zur Verfügung, die es ermöglicht, knöcherne Strukturen in CT-ähnlicher Qualität in einem Datenvolumen von $12 \mathrm{~cm}$ Kantenlänge abzubilden und daraus in beliebiger Schnittführung multiplanare Rekonstruktionen zu erstellen.

\section{Intraoperative dreidimensionale Bildverstärkerkontrolle}

Ziel einer an unserer Klinik durchgeführten prospektiven Studie war die Überprüfung der Reposition der Fibula in die Inzisur durch die intraoperative Anwendung der dreidimensionalen Bildgebung mit dem Iso- $\mathrm{C}^{3 \mathrm{D}}$ und dem Arcadis Orbic ${ }^{3 \mathrm{D}}$.

\section{Material und Methode}

Im Rahmen der Studie kamen der Siremobil Iso- $\mathrm{C}^{3 \mathrm{D}}$ und der Arcadis Orbic ${ }^{3 \mathrm{D}}$ der Fa. Siemens zum Einsatz. Während einer motorisierten Orbitalbewegung von $190^{\circ}$ um ein Isozentrum erstellen die $3 \mathrm{D}$ Bildwandler 100 Einzelprojektionen in digitaler Radiographietechnik. In einem Rechenalgorithmus wurden die Einzelprojektionen geometrisch entzerrt, unter Verwendung von Kalibrationsmatri-

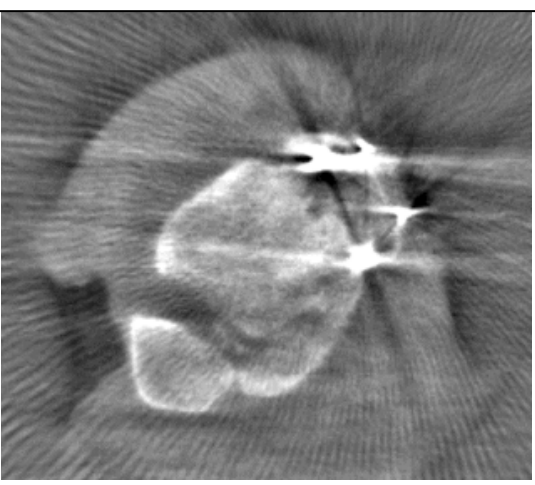

Abb. $9 \Delta$ Nicht regelrechte Einstellung bei interponiertem Fragment

zen gefaltet und ein Datenkubus von etwa $12 \mathrm{~cm}$ Kantenlänge generiert. Die Auflösung innerhalb desselben beträgt o, $45 \mathrm{~mm}$ (- Abb.3).

Im Zeitraum vom 01.06.2003-30.11.2004 wurden 15 Pronations-Eversions-Frakturen mit intraoperativer Stellschraubentransfixation und 8 Revisionseingriffe bei computertomographisch gesicherter Fehlstellung des Außenknöchels eingeschlossen. In allen Fällen wurde nach Reposition und Osteosynthese die Stabilität des Syndesmosenkomplexes überprüft.

Im Anschluss wurde die Stellschraube in oben beschriebener Technik eingebracht. Osteosynthese und Stellung der Malleolengabel (• Abb.4) wurden in der ${ }_{2}$ D-Bildwandlertechnik in a.-p. Projektion, in $20^{\circ}$ Innenrotation sowie streng seitlich überprüft. Offensichtliche Fehlstellungen wurden sofort korrigiert. Zeigte die $2 \mathrm{D}$-Kontrolle ein zufrieden stellendes Repositionsergebnis, wurde die $3 \mathrm{D}$-Bildwandlerkontrolle durchgeführt.

Reposition und Stellung der Malleolengabel wurden in multiplanaren Rekonstruktionen in axialer, koronarer und sagittaler Schichtführung dargestellt. In den koronaren Rekonstruktionen wurde die Länge der Fibula (• Abb. 5), in den axialen Darstellungen ihre Stellung in der Inzisur ( Abb. 6) und die Torsion des Außenknöchels (- Abb. 7) beurteilt. In den sagittalen Rekonstruktionen wurde ggf. die Reposition des hinteren Volkmann-Dreiecks überprüft.

\section{Ergebnisse}

Die intraoperative dreidimensionale Bildgebung war in allen Fällen möglich, Darstellung und Kontrast waren zur Beantwortung aller Fragen ausreichend. Der zu- 
Tab. 1 Fehlpositionen der Primäreingriffe

\begin{tabular}{|ll|}
\hline Fehlstellung & Anzahl ( $\boldsymbol{n}$ ) \\
Verschiebung in a.-p. Richtung & 7 \\
\hline Rotation & 2 \\
\hline Länge & 2 \\
\hline Gabel erweitert & 1 \\
\hline Korrektur Volkmann-Fragment & 1 \\
\hline Interponiertes Fragment & 1 \\
\hline
\end{tabular}

Tab. 2 Fehlpositionen der Revisionseingriffe

Fehlstellung Anzahl ( $n)$

Verschiebung in a.-p. Richtung 3

Rotation

Länge 1

Korrektur Volkmann-Fragment

sätzliche Zeitbedarf betrug für Lagerung, Bildgebung und Beurteilung im Durchschnitt 4,4 min.

Revisionsbedürftige Befunde fanden sich bei den Primäreingriffen in 6 von 15 Fällen. In 2 Fällen persistierte nach der initialen Korrektur weiterhin ein revisionsbedürftiger Befund, sodass eine nochmalige Korrektur erforderlich wurde. In - Tab. 1 sind alle Richtungen der Fehlpositionen aufgelistet.

Bei den Revisionseingriffen musste in 5 von 8 Fällen korrigiert werden, davon in 2 Fällen bei noch immer unbefriedigender Stellung ein weiteres $\operatorname{Mal}$ (• Tab. 2).

In $-\mathrm{Abb} .8$ und 9 sind Beispiele für intraoperativ korrekturbedürftige Stellungsbefunde gezeigt.

\section{Diskussion}

Sprunggelenkfrakturen mit Ruptur der tibiofibularen Syndesmose und konsekutiver Instabilität der Malleolengabel bedürfen neben einer exakten Reposition der Fraktur einer Revision der Syndesmose. Verbleibende Instabilitäten und Fehlstellungen des Außenknöchels stellen einen Präarthrosezustand dar.

Eine alleinige Naht der rupturierten und leicht zugänglichen vorderen Syndesmosenanteile ist biomechanisch nicht ausreichend. Für die Stabilität von ausschlaggebender Bedeutung sind die kurzen interossären Bandanteile. Die fibulotibiale Transfixation mit einer trikortikalen Stellschraube in der Technik nach Henkemeyer et al. [4] gilt als Goldstandard. Auch bei korrekter Anwendung der Technik sind jedoch Fehlstellungen des Außenknöchels nicht auszuschließen. Diese können durch postoperative computertomographische Kontrollen aufgedeckt werden [1], die allerdings mit einem nicht unerheblichen organisatorischen Aufwand einhergehen. Die notwendigen Revisionen bedeuten Zweiteingriffe und beinhalten zusätzliche Risiken.

Die intraoperative $\mathrm{BV}$-gestützte $3 \mathrm{D}$ Diagnostik mit dem Siremobil Iso- $\mathrm{C}^{3 \mathrm{D}}$ und dem Arcadis Orbic ${ }^{3 \mathrm{D}}$ erreicht am Sprunggelenk eine CT-ähnliche Qualität der Darstellung. Die Auflösung von etwa $0,45 \mathrm{~mm}$ ist ausreichend, um verbliebene Fehlstellungen und Gelenkstufen aufzudecken. Die integrierte Bildbearbeitung ermöglicht die Bestimmung von Winkelgraden und Abständen. Da der generierte Datensatz isotrop ist, ist eine gleichzeitige Abbildung eines Eichkörpers nicht erforderlich. Die beliebige Lage der Schnittführung ermöglicht die exakte Einstellung der Ebenen.

Unsere Serie offenbarte, dass auch bei exakter Anwendung der vorgeschlagenen Technik zur Stellschraubenplatzierung die Gefahr von Abweichungen des Außenknöchels in allen Raumebenen besteht. In unserer Studie bedeutete dies Revisionen in 2/5 der Primäreingriffe. Bei den Revisionseingriffen lag die Rate der nicht idealen Stellungen bei 5/8. Die höhere Rate der Revisionen liegt hier an der erschwerten intraoperativen Orientierung aufgrund fehlender anatomischer Landmarken nach länger zurückliegenden Voroperationen.

\section{Fazit}

In unserer Klinik wurde die intraoperative dreidimensionale Bildgebung bei Sprunggelenkverletzungen mit Syndesmoseninstabilität oder Beteiligung der Gelenkflächen zum Standard erhoben.

\section{Korrespondierender Autor}

\section{Dr. J. von Recum}

BG-Unfallklinik, Unfallchirurgische Klinik,

Universität Heidelberg

Ludwig-Guttmann-Straße 13,

67071 Ludwigshafen

recum@bgu-ludwigshafen.de

Interessenkonflikt. Es besteht kein Interessenkonflikt. Der korrespondierende Autor versichert, dass kei- ne Verbindungen mit einer Firma, deren Produkt in dem Artikel genannt ist, oder einer Firma, die ein Konkurrenzprodukt vertreibt, bestehen. Die Präsentation des Themas ist unabhängig und die Darstellung der Inhalte produktneutral.

\section{Literatur}

1. Beck M, Mittlmeier T (2004) Spezielle Probleme und Spätfolgen nach OSG-Fraktur. Trauma Berufskrankh 6 : S408-S416

2. Burns WC, Prakash K, Adelaar R et al. (1993) Tibiotalar joint dynamics: indications for the syndesmotic screw a cadaver study. Foot Ankle 14:153-158

3. Grass R, Herzmann K, Biewener A et al. (2000) Verletzungen der unteren tibiofibularen Syndesmose. Unfallchirurg 103: 520-532

4. Henkemeyer H, Püschel R, Burri C (1975) Experimentelle Untersuchungen zur Biomechanik der Syndesmose. Langenbecks Arch Chir [Suppl] 1975: 369

5. Inman V (1976) The joints of the ancle. Williams \& Wilkins, Baltimore

6. Kaye RA (1989) Stabilization of ankle syndesmosis injuries with a syndesmosis screw. Foot Ankle 9: 290293

7. Lauge-Hansen N (1948) Fractures of the ankle. Analytic-historic survey as the basis of new experimental, roentgenologic and clinical investigation. Arch Surg 56: 259-317

8. McBryde A, Chiasson B, Wilhelm A et al. (1997) Syndesmotic screw placement: a biomechanical analysis Foot Ankle Int 18: 262-266

9. Müller M, Nazarian S, Koch P (1987) Classification AO des fractures. Springer, Berlin Heidelberg New York

10. Ochs U, Winter E, Weise K (2001) Malleolenfrakturen. Trauma Berufskrankh 3: 338-343

11. Özokyay L, Muhr G, Kutscha-Lissberg F (2004) Anerkannte Indikationen zur konservativen Frakturbehandlung. Trauma Berufskrankh [Suppl 1] 6: S76-S78

12. Peter RE, Harrington RM, Henley MB et al. (1994) Biomechanical effects of internal fixation of the distal tibiofibular syndesmotic joint: comparison of two fixation techniques. J Orthop Trauma 8: 215-219

13. Ramsey P Hamilton W (1976) Changes in tibiotalar area of contact caused by lateral talar shift. J Bone Joint Surg Am 58: 356-357

14. Sasse M, Nigg B, Stefanyshyn D (1999) Tibiotalar motion - effect of fibular displacement and deltoid ligament transsection: in vitro study. Foot Ankle Int 20: 733-737

15. Stromsoe K, Hoqevold H, Skjeldal S et al. (1995) The repair of a ruptured deltoid ligament is not necessary in ankle fractures. J Bone Joint Surg Br 77: 920-921

16. Thordarson DB, Motamed S, Hedman T et al. (1997) The effect of fibular malreduction on contact pressures in an ankle fracture malunion model. J Bone Joint Surg Am 79: 1809-1815

17. Weber $B$ (1981) Lengthening osteotomy of the fibula to correct a widened mortice of the ankle after fracture. Int Orthop 4: 289-293

18. Weber B (1996) Verletzungen des oberen Sprunggelenkes. In: Weber B (Hrsg) Aktuelle Probleme in der Chirurgie, Bd 3. Huber, Bern Stuttgart Toronto

19. Xenos JS, Hopkinson WJ, Mulligan ME et al. (1995) The tibiofibular syndesmosis. Evaluation of the ligamentous structures, methods of fixation, and radiographic assessment. J Bone Joint Surg Am 77: 847-856

20. Zwipp H (1994) Chirurgie des Fußes. Springer, Berlin Heidelberg New York

21. Zwipp H, Hoffmann R, Thermann H et al. (1991) Rupture of the ankle ligaments. Int Orthop 15: 245-249

22. Zwipp H, Rammelt S, Grass R (2002) Ligamentous injuries about the ankle and subtalar joints. Clin Pediatr Med Surg 19: 195-229 